\title{
Uma aplicação do guia PMBOK na gestão de projetos de software
}

\author{
Sidgley Camargo de Andrade ${ }^{1}$ \\ Tania Fatima Calvi Tait ${ }^{2}$
}

\begin{abstract}
Resumo: Este artigo apresenta um relato de experiência de gestão de projetos de software para elaborar um plano de gerenciamento baseado nas gerências de recursos humanos, custos e riscos, seguindo as recomendações do guia de boas práticas do modelo processual do Project Management Institute (PMI). O plano de gerenciamento de projeto de software proposto refere-se ao desenvolvimento de um sistema de informação na pós-graduação, em termos de mestrado, em uma universidade pública.
\end{abstract}

Palavras-chave: Custos. Gestão de projetos de software. Recursos humanos. Riscos.

\begin{abstract}
This paper addresses an experience of software project management to develop a plan based on management of human resources, costs and risks on context of Project Management Institute (PMI). The software project management plan proposed refers to the development of an information system in a Public University specifically in the post-graduation.
\end{abstract}

Keywords: Costs. Human resources. Risks. Software project management.

\section{Introdução}

A área de gestão de projetos de software (GPS) fortaleceu-se ao longo dos anos em razão da necessidade de garantir a qualidade e o sucesso dos projetos de software. Ao aliar conceitos clássicos da área de administração, tais como planejar, coordenar, organizar e controlar, com elementos específicos da área de software, a área de GPS configura-se como multidisciplinar e integradora de aspectos tanto organizacionais como técnicos.

Por sua vez, o processo de desenvolvimento de software é formado por uma série de atividades técnicas e organizacionais que envolvem metodologias e ferramentas para elaborar, planejar, controlar e executar as atividades do projeto de desenvolvimento de software. As atividades técnicas englobam desde as metodologias de desenvolvimento até a infraestrutura do ambiente; as atividades organizacionais vão desde o atendimento às necessidades da organização até o relacionamento com os recursos humanos envolvidos em todo o ciclo de vida do projeto [4].

Diversos modelos de gerenciamento são aplicados como referência nos mais diversos segmentos [3], tais como setor público e privado, comercial e industrial, todavia, cada modelo possui suas características e especificidades metodológicas que contribuem para um gerenciamento efetivo e que garantem o sucesso do projeto.

Este trabalho apresenta um relato de experiência de uma proposta de plano de gerenciamento de projeto focado em recursos humanos, custos e riscos, seguindo as recomendações do guia de boas práticas do modelo processual do Project Management Institute (PMI) [8]. Os processos definidos e a seleção das ferramentas e

\footnotetext{
${ }^{1}$ Programa de Pós-Graduação em Ciência da Computação, UEM, Maringá (PR) - Brasil

\{sidgley@gmail.com\}

${ }^{2}$ Departamento de Informática da Universidade Estadual de Maringá, UEM, Maringá (PR) - Brasil

\{tait@din.uem.br\}
}

http://dx.doi.org/10.5335/rbca.2012.1796 
técnicas foram aplicados no desenvolvimento de um sistema de informação (SI) para a Secretaria do Programa de Pós-Graduação em Ciência da Computação (SEC-PCC) de uma universidade pública.

Este artigo está estruturado da seguinte forma: a seção 2 trata sobre gerenciamento de projeto de software e aborda o guia do PMI; na seção 3 é apresentada a metodologia de desenvolvimento da pesquisa; na seção 4 é proposto o plano de gerenciamento do projeto de software; na seção 5 são apresentadas as lições aprendidas; e na seção 6 as considerações finais para gerenciar projetos de software no contexto da secretaria da pós-graduação em uma universidade pública.

\section{Gerenciamento de projeto de software}

O gerenciamento de um projeto de software é o processo de tomar decisões que envolvem o uso de recursos, tanto materiais como humanos, para coordenar, controlar e realizar atividades, temporárias, com o objetivo de fornecer um resultado [1] [4]. A integração desses recursos em um contexto específico de desenvolvimento de software não é uma tarefa fácil e exige uma gestão e esforço coordenado das pessoas envolvidas [1].

O GPS surgiu com base no gerenciamento de projeto (GP) de outras áreas já maduras, cujas técnicas foram adaptadas em um sequência de estágios que envolvem os aspectos e questões do processo de desenvolvimento de um projeto de software, desde o estabelecimento inicial dos conceitos até o artefato de programação [10] [2]. Nesse contexto, a gestão de projetos difere das outras áreas por possuir características e fatores que dificultam ainda mais as atividades do gerenciamento, tais como mudanças tecnológicas, qualificação específica dos recursos humanos, necessidades específicas de recursos materiais, habilidades multidisciplinares e dificuldades de mensurar o software [2]. Para mitigar os impactos desses fatores é necessária a adoção de modelos e técnicas específicas para gerenciar projetos de software.

Uma série de modelos de gestão de projetos e serviços de software foi elaborada nos últimos anos. Alguns desses modelos são originais e outros são derivados e/ou evoluídos de outros modelos [3]. Para o objeto de estudo deste trabalho foram aplicadas as técnicas e ferramentas do guia de boas práticas Project Management Body of Knowledge (PMBoK), definidas pelo PMI [8].

O PMBoK é um guia reconhecido de gestão de projetos que descreve normas, métodos, processos e práticas estabelecidas para gerenciar projetos de qualquer natureza [8]. Os principais objetivos do PMBoK (2008) são as boas práticas da aplicação das ferramentas e técnicas para aumentar as chances de sucesso de um projeto e o vocabulário comum utilizado pelos profissionais da área de gerenciamento de projetos. Mesmo não sendo uma metodologia de gerenciamento de projetos, o PMBoK incorpora processos e atividades que suprem as necessidades de todas as etapas ou fases do ciclo de vida de um projeto, sendo estas definidas por Keelling (2002) como conceituação, planejamento, implementação e conclusão. Dessa forma é plausível e beneplácito a adoção do PMBoK no planejamento dos recursos humanos, riscos e custos. Outro fator que impulsionou a utilização deste corpo de conhecimento é sua característica genérica e aplicada a todos os tipos de projetos [8], inclusive projetos de desenvolvimento de software.

\section{Metodologia de desenvolvimento da pesquisa}

O processo de desenvolvimento da pesquisa foi composto pelas etapas de fundamentação teórica sobre o plano de gerenciamento de projeto, contextualização do estudo de caso e elaboração e apresentação do plano de gerenciamento de projeto de software.

\subsection{Plano de gerenciamento de projeto}

As boas práticas definidas no guia PMBoK aplicam o gerenciamento de projeto por meio da integração de áreas de conhecimento, denominadas gerências de projetos. As gerências são compostas por uma série de processos, e incluem, entre outros, aquisição, escopo, qualidade, cronograma, orçamento, recursos humanos e riscos [8]. 
A complexidade e necessidade de cada projeto definem quais gerências e processos devem ser aplicados para gerenciar as atividades do projeto. No caso específico desse projeto optaram-se pelas gerências de recursos humanos, custos e riscos em razão de dois fatores: a análise do tamanho e da complexidade do problema; e a avaliação do que preocupava os usuários do sistema tanto em nível de coordenação como operacional.

- Tamanho e complexidade do problema: a partir da análise e especificação do sistema observou-se a necessidade do desenvolvimento de apenas um módulo simples e específico com poucos casos de uso, sendo estes gerenciar acesso, inscrever no programa (programa de mestrado), realizar matrícula, solicitar convalidação, controlar bolsas e emitir diário de classe. Ressalta-se que o desenvolvimento e o acompanhamento das partes do sistema ocorrerão dentro das instalações da universidade e utilizarão os recursos disponíveis pelo programa de mestrado.

- Preocupação dos usuários: por se tratar de um projeto pequeno e específico de um setor, a baixa burocratização é uma exigência dos usuários, bem como a necessidade de possuir e utilizar o sistema o mais breve possível. Alguns recursos serão fornecidos e gerenciados pelo próprio programa de mestrado, tais como infraestrutura e materiais de experiente.

O plano de gerenciamento de recursos humanos (PGRH) é um processo do gerenciamento de recursos humanos e define uma série de processos e técnicas para identificar, selecionar e alocar pessoal para a equipe do projeto [8].

O plano de gerenciamento de custos (PGCST) inclui os processos que visam estimar, orçar e controlar os custos dos recursos necessários para terminar o projeto dentro do orçamento aprovado [8] [9].

O plano de gerenciamento de riscos (PGRSC) é o processo de definição de como conduzir as atividades de gerenciamento de riscos de um projeto, garantindo que o grau, o tipo e a visibilidade dos riscos sejam identificados, analisados e controlados [8] [6].

A gerência de escopo inclui os processos de elaboração do escopo, da coleta de requisitos e da criação da estrutura analítica do projeto (EAP), além de servir como entrada de dados para a elaboração dos planos de recursos humanos, custos e riscos [8]. No entanto, não foi elaborado um plano de gerenciamento para essa área de conhecimento por existir um documento de requisito que especificava o escopo e as atividades do projeto de desenvolvimento do SI.

\subsection{Contextualização do estudo de caso}

A SEC-PCC, vinculada ao programa de Pós-Graduação em Ciência da Computação (PCC) da universidade, foi instrumento para propor um plano de gerenciamento de projeto referente à proposta de desenvolvimento de um SI para melhorias no processo de gestão da informação.

A SEC-PCC é responsável por diversas atividades, entre outras, o registro dos alunos e professores, convênios e parcerias, controle de bolsas e interação com outros setores da universidade. Em 2009 foram identificados processos funcionais da SEC-PCC que poderiam ser otimizados, mas que exigiriam, contudo, o desenvolvimento de um software específico.

O desenvolvimento do SI proposto tem o objetivo de melhorar a informatização do processo de gerência do PCC desde a inscrição do candidato até a sua defesa ao final do curso, enfatizando o controle e a distribuição de bolsas de estudo disponibilizadas pelos órgãos de fomento.

A análise e a elaboração do documento de requisitos foram realizadas no segundo semestre de 2009 e nela constam os requisitos funcionais e não funcionais, a contextualização dos casos de uso e diagramas da linguagem de modelagem unificada (UML).

As melhorias propostas pelo desenvolvimento do SI englobam, entre outros, os processos de integração com a Diretoria de Assuntos Acadêmicos (DAA) e a Pró-Reitoria de Pesquisa e Pós-Graduação (PPG), integração de cadastros (SEC-PPC e DAA), e eficiência na recuperação e localização das informações dos alunos e professores do PCC.

Visando à continuidade da iniciativa do desenvolvimento do SI e ao incentivo a pesquisa, o Departamento de Informática (DIN) sugeriu a elaboração de um plano de gestão e execução do projeto de desenvolvimento do 
software. No segundo semestre de 2010 realizou-se um estudo do cenário e propôs-se um plano de gerenciamento concentrado nas gerências de recursos humanos, riscos e custos.

\subsection{Elaboração e apresentação do plano de gerenciamento de projeto de software}

Para alcançar o objetivo do trabalho proposto, qual seja a elaboração de um plano de gerenciamento de projeto de software, foram realizadas pesquisas in loco por meio da análise do documento de requisitos, de observações no âmbito da SEC-PCC e de entrevistas com a secretária da SEC-PCC.

O documento de requisitos proporcionou uma compreensão holística do processo, ao passo que as observações e as entrevistas permitiram compreender com maior clareza as necessidades da SEC-PCC. Para entender todo o contexto e definir a estratégia do GPS, os resultados foram interpretados e verificados no contexto do PCC. O estudo foi realizado na SEC-PCC entre os meses de setembro e novembro de 2010.

\section{Plano de gerenciamento de projeto de software proposto}

A elaboração do Plano de Gerenciamento de Projeto de Software (PGPS), proposto para o desenvolvimento do SI, concentra-se nas áreas de gerência de recursos humanos, gerência de riscos e gerência de custos. As outras gerências que compõem o guia de boas práticas do PMBoK foram desconsideradas neste trabalho ou pela característica do projeto ou por estarem contidas nas atividades gerenciais do próprio programa de mestrado.

Foram desenvolvidos três planos de gerenciamento, um para cada processo de gerência. O plano de gerenciamento especifica as técnicas, ferramentas e interações aplicadas em cada área de conhecimento. $\mathrm{O}$ conjunto dos planos individuais define o PGPS (Fig. 1).

Os planos individuais (recursos humanos, custos e riscos) foram elaborados conforme a especificação das boas práticas de aplicabilidade das ferramentas e técnicas do PMBoK. Os planos são descritos nas subseções seguintes.

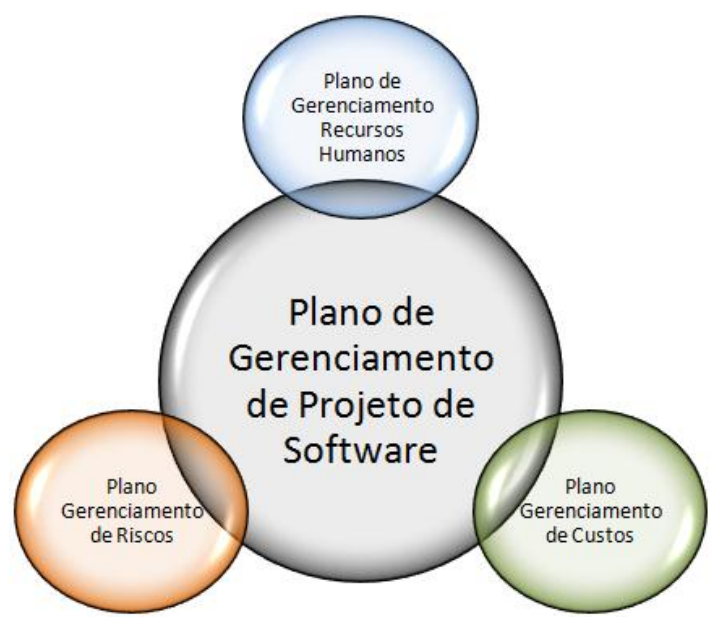

Figura 1: Plano de gerenciamento de projeto de software

\subsection{Plano de gerenciamento de recursos humanos (PGRH)}

Após análise da estrutura organizacional e stakeholders do projeto, foram selecionadas as técnicas e as ferramentas para compor o Plano de Gerenciamento de Recursos Humanos (PGRH). A parceria entre o PCC e o Núcleo de Processamento de Dados da universidade (NPD) é o cerne estratégico do planejamento proposto. Essa estratégia simplifica e agiliza o processo de identificação e alocação de recursos humanos. 
O NPD possui profissionais capacitados e com o perfil que satisfaz os critérios estabelecidos em comum acordo entre a gerência e o patrocinador do projeto (PCC). A experiência no setor público, o conhecimento da estrutura de uma universidade pública, a pesquisa e a pós-graduação são os principais critérios exigidos.

A relação hierárquica e as responsabilidades dos recursos humanos selecionados foram determinadas com as técnicas da estrutura analítica organizacional (EAO), do organograma textual, e da matriz de responsabilidades (MR).

Por se tratar de uma universidade pública e o envolvimento de alguns setores, a estrutura organizacional foi definida para distribuir as responsabilidades e estabelecer delimitadores entre os envolvidos no projeto, visando o comprometimento, a confiança e o respeito.

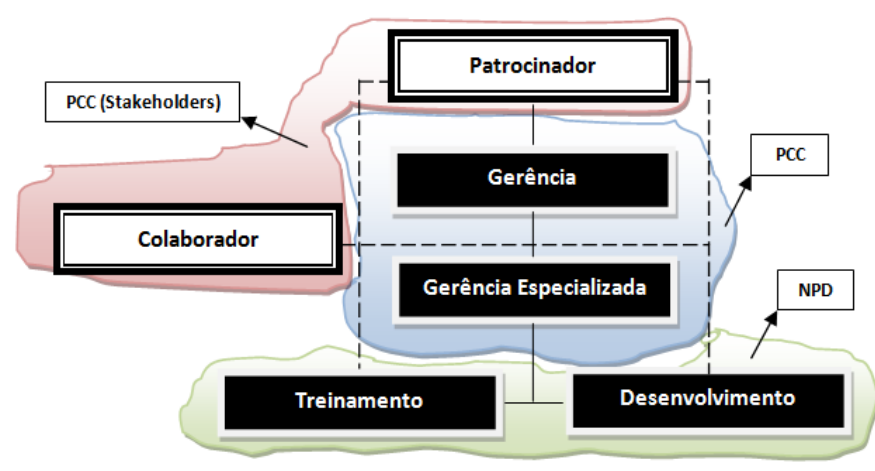

Figura 2: Estrutura analítica organizacional do PGRH

A EAO é uma representação gráfica hierárquica organizada de acordo com as relações departamentais dos stakeholders. No contexto do projeto, a estrutura das relações foi classificada em interna e externa conforme contexto da SEC-PCC. A equipe de gerenciamento, desenvolvimento e treinamento (integrantes do PCC e NPD) compõem a estrutura externa, ao passo que o patrocinador (responsável do PCC) e os colaboradores (SEC-PCC, DAA, DIN e PPG) compõem a estrutura interna do projeto.

Apesar da definição da EAO, a hierarquia organizacional dentro do projeto não é rígida e permite a interação direta entre os membros, facilitando a comunicação e a troca de informações em prol do sucesso do projeto. Essa estrutura é possível ao passo da flexibilidade do DIN e da natureza dos setores envolvidos, cuja independência política, a interação e a comunicação vigoram.

Tabela 1: Organograma textual do PGRH

\begin{tabular}{lll}
\hline Papel & Cargo & Responsabilidade/Autoridade \\
\hline Gerência & Gerente de projeto & $\begin{array}{l}\text { Responsável pelo projeto. Gerencia o projeto no sentido } \\
\text { de atingir sua completude de qualidade, prazo e custo. }\end{array}$ \\
\hline $\begin{array}{l}\text { Gerência } \\
\text { especializada }\end{array}$ & $\begin{array}{l}\text { Líder de } \\
\text { treinamento }\end{array}$ & $\begin{array}{l}\text { Responsável pelo treinamento da equipe do projeto e dos } \\
\text { usuários. Subordinado ao gerente de projetos. }\end{array}$ \\
\cline { 2 - 4 } & $\begin{array}{l}\text { Líder de } \\
\text { desenvolvimento }\end{array}$ & $\begin{array}{l}\text { Responsável pelo gerenciamento da equipe de } \\
\text { desenvolvimento e qualidade do produto. Responde pela } \\
\text { elicitação e documentação de requisito. Subordinado ao } \\
\text { gerente de projetos. }\end{array}$ \\
\cline { 2 - 4 } Colaborador & - & $\begin{array}{l}\text { Auxiliar e fornecer dados do projeto a gerência } \\
\text { especializada. Os colaboradores não estão subordinados a } \\
\text { nenhum papel, flexibilizando a comunicação entre os } \\
\text { envolvidos no projeto. }\end{array}$ \\
\cline { 2 - 4 } Treinamento & Analista & $\begin{array}{l}\text { Executor dos treinamentos gerenciados pelo líder de } \\
\text { treinamento. }\end{array}$ \\
\cline { 2 - 4 } Desenvolvimento & Analista & Desenvolvedor e codificador dos projetos de software. \\
\hline
\end{tabular}

A estrutura hierárquica em nível de papel é representada pela EAO (Fig. 2), e a descrição das atividades, responsabilidades, cargo e subordinação de cada papel pelo organograma textual (Tab. 1). Como não foi possível 
identificar os recursos humanos disponível do NDP, o organograma textual também foi detalhado em nível de papel. É importante esclarecer que existe a possibilidade de rearranjar as atividades atuais do NPD para alocar novas tarefas aos colaboradores, contudo essa atividade é de responsabilidade do próprio NDP.

A MR ilustra as conexões entre pacotes de trabalho ou atividades e os membros da equipe do projeto [8] [7]. A MR proposta no PGRH (Tab. 2) é uma adaptação da MR em formato RACI apresentada no PMBoK, e sua estrutura foi baseada na estrutura analítica do projeto.

Tabela 2: Matriz de responsabilidades do PGRH

\begin{tabular}{|c|c|c|c|c|c|c|c|c|c|c|}
\hline \multirow[t]{2}{*}{ Nome } & \multirow[t]{2}{*}{ Papel } & \multirow[b]{2}{*}{ 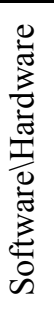 } & \multirow[b]{2}{*}{ 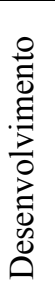 } & \multirow[b]{2}{*}{ 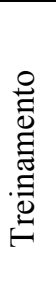 } & \multirow[b]{2}{*}{ 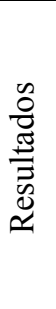 } & \multicolumn{5}{|c|}{ Gerenciamentos } \\
\hline & & & & & & $\begin{array}{l}\circ \\
\stackrel{2}{0} \\
0 \\
\text { ஸు }\end{array}$ & 呑 & $\stackrel{0}{\stackrel{0}{0}}$ & $\frac{1}{2}$ & $\begin{array}{l}\mathscr{0} \\
\mathscr{0} \\
\tilde{\mathscr{L}}\end{array}$ \\
\hline Fulano & Gerente & $\mathrm{I}$ & $\mathrm{I}$ & $\mathrm{I}$ & $\mathrm{R}$ & A & AR & AR & AR & AR \\
\hline Beltrano & Líder de treinamento & & $\mathrm{S}$ & $\mathrm{A}$ & $\mathrm{I}$ & $\mathrm{S}$ & $\mathrm{S}$ & $\mathrm{S}$ & $\mathrm{S}$ & $\mathrm{S}$ \\
\hline Sicrano & Líder de desenvolvimento & A & A & $\mathrm{S}$ & $\mathrm{I}$ & $\mathrm{R}$ & $\mathrm{S}$ & $\mathrm{S}$ & $\mathrm{S}$ & $\mathrm{S}$ \\
\hline PCC & Patrocinador & & $\mathrm{I}$ & $\mathrm{I}$ & $\mathrm{A}$ & & I & $\mathrm{I}$ & $\mathrm{I}$ & $\mathrm{I}$ \\
\hline DAA & Colaborador & & $\mathrm{I}$ & & $\mathrm{I}$ & & & & & \\
\hline SEC-PCC & Colaborador & & I & $\mathrm{I}$ & A & A & I & I & I & I \\
\hline PPG & Colaborador & & $\mathrm{I}$ & & I & & & & & \\
\hline $\mathrm{DIN}$ & Colaborador & & $\mathrm{I}$ & & $\mathrm{I}$ & & & & & \\
\hline NPD & Treinamento/Desenvolvedor & $\mathrm{R}$ & $\mathrm{R}$ & $\mathrm{R}$ & $\mathrm{I}$ & $\mathrm{I}$ & $\mathrm{I}$ & I & I & I \\
\hline
\end{tabular}

\subsection{Plano de gerenciamento de custos (PGCST)}

No plano de gerenciamento de custos (PGCST) delimita-se a aplicação dos processos de estimativas de custos baseado no tempo das atividades e na disponibilidade dos recursos humanos. A aplicação da técnica de revisão e avaliação de programa (PERT) possibilita a avaliação quantitativa dos custos prováveis dos recursos humanos necessários para completar as atividades do projeto dentro de um prazo estimado.

A análise empírica do tempo de desenvolvimento dos casos de uso e a da disponibilidade dos recursos humanos, realizada no PGRH, determinou a escolha da técnica PERT. Os colaboradores do NPD estão inseridos em vários projetos solicitados pela universidade pública e a precisão das estimativas de custo deve considerar variabilidades de risco, tais como o atraso no início das atividades e a permuta de colaboradores. O gráfico em rede PERT foi construído a partir dos use cases da estrutura analítica e define três medidas de tempo para gerar o custo do projeto: tempo ótimo $\left(\mathrm{t}_{\mathrm{o}}\right)$, tempo médio $\left(\mathrm{t}_{\mathrm{m}}\right)$ e tempo péssimo $\left(\mathrm{t}_{\mathrm{p}}\right)$.

As medidas são estimativas do tempo necessário para desenvolver cada use case. A Tabela 3 ilustra a análise da estimativa de tempo do plano proposto.

Tabela 3: Análise da estimativa de tempo do PGCST

\begin{tabular}{|c|c|c|c|c|c|c|c|c|}
\hline Nr. & Use case & Prec. & $\left(\mathrm{t}_{\mathrm{o}}\right)$ & $\left(t_{m}\right)$ & $\left(t_{p}\right)$ & $\left(\mathrm{t}_{\mathrm{e}}\right)$ & $\left(\approx d_{\mathrm{uc}}\right)$ & $\left(t_{f}\right)$ \\
\hline 1 & Gerenciar acesso & - & 2 & 4 & 6 & 4 & 1 & 5 \\
\hline 2 & Inscrever no programa & 1 & 1 & 2 & 4 & 2 & 1 & 3 \\
\hline 3 & Realizar matrícula & 2 & 1 & 2 & 3 & 2 & 0 & 2 \\
\hline 4 & Solicitar convalidação & 3 & 2 & 3 & 5 & 3 & 0 & 3 \\
\hline 5 & Controlar bolsas & 3 & 3 & 4 & 6 & 4 & 1 & 5 \\
\hline \multirow[t]{2}{*}{6} & Emitir diário de classe & 3 & 1 & 2 & 3 & 2 & 0 & 2 \\
\hline & Tempo ( & ana) & 10 & 17 & 27 & 17 & 3 & 20 \\
\hline
\end{tabular}


A unidade de desenvolvimento definida foi a semanal, então os valores apresentados na tabela da análise da estimativa de tempo correspondem ao número de semanas necessárias para o desenvolvimento e teste de cada use case.

Com base no PMI (2008), a estimativa do tempo esperado $\left(t_{\mathrm{e}}\right)$ é obtida usando a média ponderada das medidas $\mathrm{t}_{\mathrm{o}}, \mathrm{t}_{\mathrm{m}} \mathrm{e} \mathrm{t}_{\mathrm{p}}$. Os pesos foram distribuídos de acordo com critérios definidos pela distribuição beta PERT, e foram atribuídos, respectivamente, peso 4 para o tempo médio, peso 1 para o tempo ótimo e peso 1 para o tempo péssimo. A estimativa do tempo final $\left(\mathrm{t}_{\mathrm{f}}\right)$ foi calculada com base na estimativa do tempo esperado e seu desvio padrão $\left(\approx d_{u c}\right)$.

Os custos dos use cases (Tabela 4) são estimativas definidas pela gerência do projeto em conjunto com os analistas do NPD e baseado no resultado da estimativa do tempo final (Tab. 3), na complexidade do gráfico PERT (Fig. 3), no documento de requisitos e na experiência da gerência em projetos antigos. Para o escopo desse projeto definiu-se um custo médio de desenvolvimento de $\mathrm{R} \$ 1.000,00$ por use case.

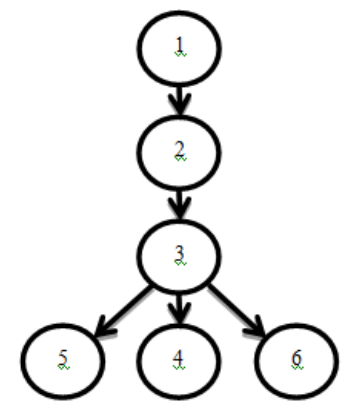

Figura 3: Gráfico PERT

A parceria com os departamentos DIN e NPD, definidos no PGRH, reduziu o custo do projeto. Custos referentes a recursos materiais, despesas variáveis (energia, telefone, materiais), entre outros, são gerenciados e fornecidos pelo NPD e pela universidade pública.

\begin{tabular}{|c|c|c|}
\hline Nr. & Use Case & $\left(\mathrm{c}_{\mathrm{uc}}\right)$ \\
\hline 1 & Gerenciar acesso & 5.000 \\
\hline 2 & Inscrever no programa & 3.000 \\
\hline 3 & Realizar matrícula & 2.000 \\
\hline 4 & Solicitar convalidação & 3.000 \\
\hline 5 & Controlar bolsas & 5.000 \\
\hline 6 & Emitir diário de classe & 2.000 \\
\hline
\end{tabular}

\subsection{Plano de gerenciamento de riscos (PGRSC)}

O escopo do projeto de desenvolvimento do software descrito no documento de análise e requisitos fornece uma percepção para identificar os principais riscos do projeto. Reuniões realizadas entre a gerência do projeto, alguns dos stakeholders (secretária do PCC) e analistas de sistema (NPD) contribuíram para identificar, classificar e definir ações de tratamento sobre os riscos do projeto.

Tabela 5: Qualificação da probabilidade de ocorrência do risco

\begin{tabular}{ll}
\hline Probabilidade & Faixa \\
\hline Muito baixa & Inferior a $10 \%$ \\
Baixa & Entre 10 e $25 \%$ \\
Média & Entre 25 e $50 \%$ \\
Alta & Entre 50 e $75 \%$ \\
Muita alta & Superior a $75 \%$ \\
\hline
\end{tabular}


Os riscos identificados foram analisados e classificados em dois aspectos, quais sejam a probabilidade de ocorrência (Tabela 6) e o efeito da incidência (Tabela 5). A Tabela 7 apresenta a identificação, a classificação e o tratamento de alguns dos riscos identificados na fase inicial do projeto. Existem outros riscos, contudo, os apresentados caracterizam o projeto e destacam-se dos demais.

O principal risco identificado no planejamento é a indisponibilidade de recursos humanos para cumprir as datas e os orçamentos. A disponibilidade dos recursos compete ao NPD e sua política de priorização de projetos pode comprometer a execução contínua das atividades, pois privilegia a área administrativa da universidade pública.

Tabela 6: Qualificação do efeito de incidência do risco

\begin{tabular}{l}
\hline Efeito \\
\hline Catastrófico \\
Sério \\
Tolerável \\
\hline
\end{tabular}

Tabela 7: Identificação, classificação e tratamento dos riscos

\begin{tabular}{|c|c|c|c|}
\hline Risco & Probabilidade & Efeito & Tratamento \\
\hline $\begin{array}{l}\text { Especificação dos use cases } \\
\text { inadequadas }\end{array}$ & Baixa & Sério & $\begin{array}{l}\text { Realizar nova elicitação de } \\
\text { requisites. }\end{array}$ \\
\hline Problemas de design & Média & Sério & Prototipação das interfaces. \\
\hline Indisponibilidade de recursos humanos & Alta & Sério & $\begin{array}{l}\text { Risco não tratado por se tratar de } \\
\text { um projeto executado em } \\
\text { paralelo pela equipe do NPD. }\end{array}$ \\
\hline Atraso na entrega dos use cases & Média & Sério & $\begin{array}{l}\text { Solicitar mais recursos humanos } \\
\text { e materiais ao NPD. }\end{array}$ \\
\hline Substituição de gerência & Baixa & Catastrófico & $\begin{array}{l}\text { Suplente deve assumir (matriz de } \\
\text { responsabilidades). }\end{array}$ \\
\hline $\begin{array}{l}\text { Problemas financeiros da organização } \\
\text { forçam reduções no orçamento do } \\
\text { projeto }\end{array}$ & Baixa & Catastrófico & Interrupção do projeto. \\
\hline Estimativas imprecisas & Baixa & Catastrófica & $\begin{array}{l}\text { Refazer o PGCST, a estimativa } \\
\text { de tempo dos use cases e o } \\
\text { PGRSC. }\end{array}$ \\
\hline Stakeholders pouco cooperativos & Baixa & Sério & $\begin{array}{l}\text { Trabalho de conscientização pela } \\
\text { gerência de treinamento. }\end{array}$ \\
\hline Problemas de infraestrutura de rede & Baixa & Tolerável & $\begin{array}{l}\text { Aguardar responsáveis pela } \\
\text { infraestrutura restabelecerem os } \\
\text { serviços. }\end{array}$ \\
\hline Rotatividade dos membros da equipe & Média & Tolerável & $\begin{array}{l}\text { Apresentar escopo do projeto e } \\
\text { realizar treinamento. }\end{array}$ \\
\hline Homologação do sistema & Média & Tolerável & $\begin{array}{l}\text { Cobrar e conscientizar os } \\
\text { usuários da necessidade da } \\
\text { homologação. Avaliar o risco de } \\
\text { atraso na entrega dos use cases. }\end{array}$ \\
\hline Aquisição de hardware e software & Baixa & Tolerável & $\begin{array}{l}\text { Solicitar ao NPD. Avaliar o risco } \\
\text { de atraso na entrega dos use } \\
\text { cases. }\end{array}$ \\
\hline
\end{tabular}




\section{Lições aprendidas}

O gerenciamento de projeto de software é uma área da Engenharia de Software (ES) que está em constante transformação. Existem vários modelos que visam elaborar, planejar, controlar e executar os projetos de softwares, todavia o uso adequado das ferramentas e técnicas se faz necessário para atingir os objetivos do projeto.

Este artigo relata a experiência da fase inicial da gestão de projeto de software para o desenvolvimento de um SI em uma universidade pública, e evidencia as recomendações do guia PMBoK na seleção dos processos, ferramentas e técnicas aplicadas na fase de planejamento das gerências de recursos humanos, custos e riscos.

O estudo realizado para elaborar o PGPS proporcionou o conhecimento em relação às necessidades e características do setor público, tais como:

- a rotatividade de pessoal;

- a alocação de pessoal em projetos;

- a demora na aquisição de equipamentos;

- o comprometimento em projetos que não visam melhorias diretas;

- a necessidade da colaboração e coordenação entre os setores da universidade pública.

Também foi possível observar alguns dos benefícios e das limitações do guia PMBoK dentro do contexto deste trabalho:

- a aplicação das ferramentas e técnicas fornecidas pelo guia satisfaz as necessidades dos gestores e são facilmente adaptadas em diferentes contextos e natureza de projetos;

- a aplicação de um planejamento geral para a gestão de projeto de software em uma universidade pública com ênfase nos planos de recursos humanos, riscos e custos;

- a não utilização do guia como metodologia de gestão de projetos de software;

- a necessidade de estabelecer todo o escopo do projeto antes de iniciá-lo.

\section{Considerações finais}

Este artigo apresentou um relato de experiência do desenvolvimento de um plano de gerenciamento de projeto de software constituído pelas gerências de recursos humanos, custos e riscos. O plano foi baseado no guia PMBoK (2008) e aplicado no desenvolvimento de um sistema de informação para a secretaria de pósgraduação em Ciência da Computação de uma universidade pública.

O estudo sobre gerenciamento de projetos de software e a elaboração do plano de gerenciamento para o setor público contribuíram no desenvolvimento de novos conhecimentos e experiências. Como recomendações sobre a forma de gerenciar o desenvolvimento de software em universidades públicas estão:

- aproveitar a infraestrutura para o funcionamento do SI proposto;

- aproveitar os recursos materiais e humanos do núcleo de tecnologia da informação;

- fortalecer a integração entre setores envolvidos;

- armazenar o conhecimento dos envolvidos para minimizar o nível de dependência e não comprometer a execução do projeto;

- organizar informações para tomada de decisão por parte da coordenação e dos órgãos universitários ligados à pesquisa;

- fomentar a implementação de sistema para tomada de decisão nos níveis táticos e estratégicos da universidade;

- implantar um sistema único de controle de secretaria para os programas de pós-graduação. 
Como trabalho futuro sugere-se a elaboração de um novo plano de gerenciamento de projeto de software que contemple a implantação e a integração dos sistemas de controle de secretaria para os programas de pósgraduação da universidade pública e a aplicação de outras gerências e ferramentas que possam apoiar o gerente de projeto no controle e coordenação das demais secretarias e setores da universidade pública. Hoje, parte dos programas de pós-graduação da universidade pública não possui um sistema adequado de gestão dos alunos e comunicação com outros programas de pós-graduação ou departamentos internos ou externos à instituição de ensino. Com a extensão do projeto inicial aos demais programas de pós-graduação aplicam-se novos conceitos de gestão de projetos, tais como programa de projetos e gerenciamento de configuração.

Como contribuição espera-se também que este trabalho dissemine e desperte o interesse dos administradores e gestores dos programas de pós-graduação na adoção e utilização de técnicas de gerenciamento de projetos, visando melhorar a qualidade e controle das atividades internas das secretarias de pós-graduação da universidade pública.

\section{Referências}

[1] CASEY, V. Virtual software team project management. Journal of the Brazilian Computer Society, Publisher: Springer, v. 16, issue 2, p. 1-14, 2010.

[2] ENAMI, Lucia; TAIT, Tania F. C.; HUZITA, Elisa H M. A project management model to a distributed software engineering environment. In: ICEIS 2006 - INTERNATIONAL CONFERENCE ON ENTERPRISE INFORMATION SYSTEMS, 2006, Papus. Anais do ICEIS 2006, 2006.

[3] FERNANDES, A. A.; ABREU, V. F. Implantando a governança de TI: da estratégia à gestão dos processos e serviços. 2. ed. Rio de Janeiro: Brasport, 2008. 444 p.

[4] HUZITA, E. H. M.; TAIT, T. F. C. Gerência de projetos de software. Escola Regional de Informática. Bandeirantes - Paraná, 2006.

[5] KEELLING, R. Gestão de projetos: uma abordagem global. São Paulo: Saraiva, 2002.

[6] LEME, L. H. R.; HUZITA, E. H. M.; TAIT, T. F. C. Strategy of risk management for a distributed software engineering environment. In: INTERNATIONAL CONFERENCE ON ENTERPRISE INFORMATION SYSTEMS - ICEIS 2007, 2007, Funchal - Portugal, 2007.

[7] MARTINS, J. C. C. Técnicas para gerenciamento de projetos de software. Rio de Janeiro: Brasport, 2007.

[8] PROJECT MANAGEMENT INSTITUTE. Um guia do conhecimento em gerenciamento de projetos - Guia PMBOK. 4. ed. Newton Square, Pennsylvania: Project Management Inst-id, 2008.

[9] SOMMERVILLE, I. Engenharia de software. 8. ed. São Paulo: Pearson Addison-Wesley, 2007.

[10] TEIXEIRA, C. A. N.; CUKIERMAN, H. L. Por que falham projetos de implantação de processos de software?. In: SIMPÓSIO BRASILEIRO DE QUALIDADE DE SOFTWARE/WOSES (Workshop Um Olhar Sociotécnico sobre a Engenharia de Software), 2007, Porto de Galinhas - PE. 3. WOSES. Rio de Janeiro: PESC/COPPE - UFRJ, 2007. p. 1-12.

[11] TAIT, Tania F. C.; PACHECO, R. C. S. Uma contribuição da área de sistemas de informação para a informática pública. In: CONGRESSO DA SOCIEDADE BRASILEIRA DE COMPUTAÇÃO, XXI Semish, XXVIII, 2001, Fortaleza - CE. Anais... Fortaleza: SBC, 2001. v. 1. p. 1-14. 